\title{
Antagonism between abscisic acid and gibberellin regulates starch synthesis and corm development in Gladiolus hybridus
}

Jingru Li ${ }^{1}$, Shanshan Seng ${ }^{1}$, Donglei Li ${ }^{1}$, Fengqin Zhang ${ }^{1}$, Yixuan Liu', Ting Yao ${ }^{1}$, Jiahui Liang ${ }^{1}$, Mingfang $\mathrm{Yi}^{1}$ and Jian Wu (1)

\begin{abstract}
Understanding corm development in flower bulbs is of importance for securing the quality of cut flowers and propagation of commercial stocks. Gladiolus is one of the most popular bulb plants worldwide. Its corm development is characterized by starch accumulation. Previous research has shown that phytohormones (especially gibberellin (GA)) are involved in tuber development. However, the relationship between abscisic acid (ABA)/GA and starch during corm development remains unclear. To gain deeper insights into the biological process of corm development, we performed a detailed anatomical characterization of different stages of corm development and analyzed phytohormone levels. Our study showed that corm development is linked to hormones (ABA and GA) and carbohydrates (sucrose and starch). Exogenous hormone treatment and silencing of endogenous hormone biosynthesis genes indicated that ABA positively regulates corm development, while GA acts as an antagonist of ABA function. A sucrose synthase gene (GhSUS2) was shown to be involved in the antagonism between ABA and GA. GhSUS2 was upregulated by ABA and downregulated by GA. The increase in the transcript level of GhSUS2 coincided with the development of corm/cormels. Silencing of GhSUS2 repressed corm development and starch accumulation. In conclusion, we propose that GhSUS2, an essential enzyme in sucrose degradation, is differentially regulated by ABA and GA and controls corm development in Gladiolus.
\end{abstract}

\section{Introduction}

A storage organ is a portion of a plant specifically modified as a reserve of energy or water. This is an evolutionary strategy that maintains plant survival and propagation from generation to generation. The storage organs of geophytes are often found underground and are modified from the root, leaf, stem, or hypocotyl. They include bulbs, tubers, corms, rhizomes, and root tubers. Storage organs serve as sources of food (e.g., potato), commercial goods (e.g., Fritillaria spp.), and decorations for gardens (e.g., Gladiolus spp.). To date, the

\footnotetext{
Correspondence: Jian Wu (jianwu@cau.edu.cn)

${ }^{1}$ Beijing Key Laboratory of Development and Quality Control of Ornamental Crops, Department of Ornamental Horticulture and Landscape Architecture, China Agricultural University, Beijing, China

These authors contributed equally: Jingru L, Shanshan Seng, Donglei Li
}

development of storage organs is poorly understood, except for potato. For wild potato species, tuberization is regulated by environmental factors, such as ambient temperature, photoperiod, and nitrogen ${ }^{1,2}$. Tuberization is stimulated under short-day conditions and promoted by low night temperatures ${ }^{1}$. Under long-day or hightemperature conditions, inhibition of tuberization is mediated by the CONSTANTS/FLOWERING LOCUS T $(\mathrm{CO} / \mathrm{FT})$ protein $^{3,4}$. In hydroponically cultivated potatoes, tuberization is induced by withdrawing nitrogen from the nutrient solution, indicating that nitrogen has an inhibitory effect on tuberization ${ }^{5}$. In the context of phytohormones, GA dominates tuberization ${ }^{6}$. The GA level decreases when the stolon tip starts to swell, and excess GA inhibits the transition from stolon to tuber ${ }^{7,8}$. GA metabolism genes (StGA20ox1 and StGA3ox2) have been

\section{(c) The Author(s) 2021}

(c) Open Access This article is licensed under a Creative Commons Attribution 4.0 International License, which permits use, sharing, adaptation, distribution and reproduction cc) in any medium or format, as long as you give appropriate credit to the original author(s) and the source, provide a link to the Creative Commons license, and indicate if changes were made. The images or other third party material in this article are included in the article's Creative Commons license, unless indicated otherwise in a credit line to the material. If material is not included in the article's Creative Commons license and your intended use is not permitted by statutory regulation or exceeds the permitted use, you will need to obtain permission directly from the copyright holder. To view a copy of this license, visit http://creativecommons.org/licenses/by/4.0/. 
shown to be involved in tuberization ${ }^{9,10}$. Cytokinins and jasmonic acid (JA) positively induce tuberization ${ }^{11,12}$. Aside from insights into the regulation of tuberization in potato, the physiological and molecular mechanisms of storage organ development in other species are poorly studied. Recently, by transcriptome and physiological analyses, a GA inhibitor was shown to stimulate the shoot-to-bulblet transition of lily in vivo, and starch was found to be a fundamental compound in this process ${ }^{13}$. However, direct genetic evidence of the role of hormones in bulb development remains elusive.

As the principal carbohydrate in storage organs, starch plays crucial roles in the development of storage organs, such as tubers and seeds ${ }^{14-16}$. Starch is an insoluble glucan comprised of amylopectin, amylose, and two polymers of glucose $^{17}$. Sucrose is loaded from photosynthetic organs (e.g., leaves) and unloaded in nonphotosynthetic organs (i.e., sink organs) where sucrose is converted to starch for long-term storage in amyloplasts. Upon arriving in sink organs, sucrose is transported into sink cells via transporters or plasmodesmata ${ }^{18,19}$. In the cytosol, sucrose can be hydrolyzed by cytosolic invertase (CIN) to fructose and glucose or cleaved by sucrose synthase (SUS) to fructose and uridine diphosphate glucose (UDP-G). In the latter pathway, UDP-G can be further transformed to adenosine diphosphate glucose (ADP-G) by UGPase and AGPase $^{20}$. ADP-G is the main substrate for starch biosynthesis in angiosperms. The glucosyl moiety is added to existing glucan chains by starch synthases ${ }^{17}$. Starch synthesis is regulated by phytohormones. In maize and potato, GA inhibits starch accumulation, while its inhibitor (chlorocholine chloride) significantly increases starch content ${ }^{21,22}$. In rice internodes, overexpression of ethylene receptors (ETHYLENE RESPONSE2/ETR3) induces the accumulation of starch granules ${ }^{23}$.

SUS is a glycosyltransferase that catalyzes the reversible transfer of a glucosyl moiety between fructose and UDP$\mathrm{G}^{24}$. UDP-G is used to biosynthesize starch or cellulose in plants $^{25}$. Selective phosphorylation of sucrose synthase isoforms results in enhanced sucrose degradation ${ }^{26}$. SUS promotes vegetative growth, early flowering, plant biomass accumulation, and the response to low oxygen ${ }^{27-29}$. Moreover, SUS decomposes sucrose in sink organs, generating a sucrose gradient flow from source to sink, i.e., the flow provides pressure for the transport of sucrose from the phloem to the sink and ensures the continuous supply of sucrose to the sink in potato. Reducing SUS activity in potato results in reduced tuber dry weight and a lower content of $\operatorname{starch}^{30}$.

Although much is known about sink organs in model plants, it has become clear that research on model plants alone will not provide adequate information for the improvement of starch accumulation in all plant species. Indeed, factors controlling starch metabolism differ among species and organs ${ }^{17}$. Therefore, it is worthwhile to study the role of starch in the development of storage organs in nonmodel plants. Gladiolus is one of the most widely planted geophytes worldwide, and its corm is the only plant stock and propagation tissue used for commercial cultivation. Corm degradation, which leads to decreased cut flower production and propagation yield, is one of the most severe problems in Gladiolus cultivation. The gladiolus corm is a specialized underground organ consisting of an enlarged stem axis with distinct nodes and internodes and enclosed by dry, scale-like leaves (tunics). A new corm is generated each growing season over the mother corm. Meanwhile, cormels are produced at the tips of branched stolons that develop from buds located at the base of the new corm $^{31}$. Corm development is tightly related to carbohydrates. During the rapid expansion of corm and cormels, starch and sugar levels increased sharply ${ }^{32}$. Recently, we have shown that induction of starch metabolic genes (the small/large subunit of ADP-glucose pyrophosphorylase; GhAGPS1/ GhAGPL1) stimulates corm development and cormel numbers $^{33,34}$. Additionally, JA could promote corm expansion, and the expression pattern of GhLOX1 (LIPOXYGENASE), a key JA biosynthesis gene, coincides with corm development ${ }^{35}$. Whether other hormones participate in corm development and how hormones regulate carbohydrates in corms are still not clear.

Here, we show that the levels of starch and endogenous ABA increase sharply during the transition from stolon to cormel, while the $\mathrm{GA}_{3}$ level decreases. Interestingly, exogenous $\mathrm{ABA}$ promotes corm weight and cormel numbers, and $\mathrm{GA}_{3}$ represses the stolon-to-cormel transition. Furthermore, decreasing the ABA level in the corm by silencing GhNCED dramatically inhibited starch accumulation and the expansion of corms, which was the opposite of the phenotype observed in GhGA20oxsilenced corms. We show that GhSUS2 expression is induced by $\mathrm{ABA}$ but inhibited by $\mathrm{GA}_{3}$. GhSUS2 is expressed mainly in sink organs (corms and cormels) and upregulated during corm development. Silencing GhSUS2 in corms impairs corm development. Hence, we propose that ABA and GA play antagonistic roles in corm development by differentially regulating GhSUS2 expression and starch synthesis in Gladiolus.

\section{Results}

Endogenous $A B A$ and GA levels, along with starch accumulation, change dramatically during Gladiolus cormel development

Early cormel development in Gladiolus can be divided into 5 stages (Fig. 1A): stolons, with a tip that is not swollen (I); pale cormels (II; $0-5 \mathrm{~mm}$ in diameter); yellow cormels (III; $5-7 \mathrm{~mm}$ in diameter); expanded cormels (IV; $7-9 \mathrm{~mm}$ in diameter) and cormels that become planting 

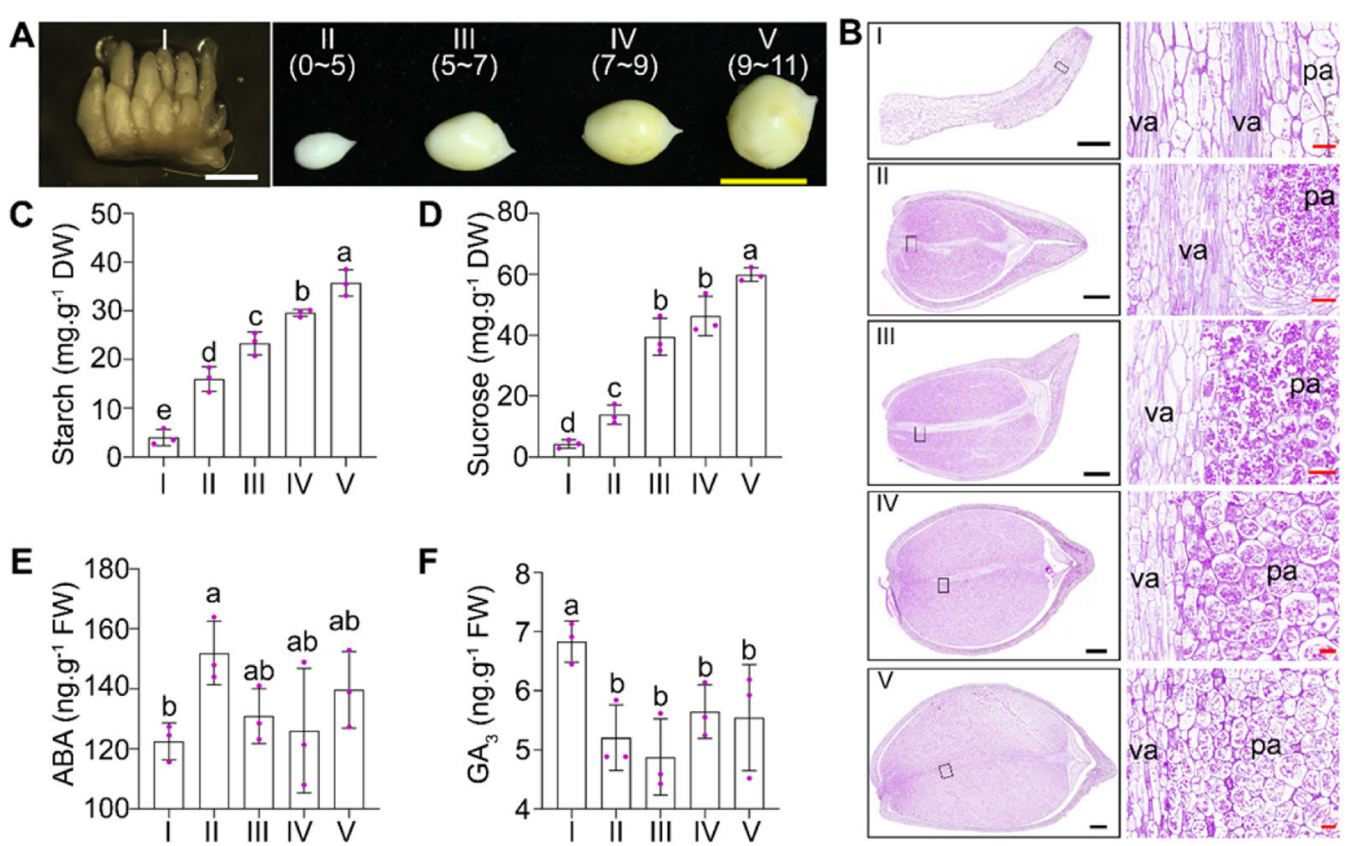

Fig. 1 Carbohydrate and endogenous hormone levels change dramatically during Gladiolus cormel development. A Early cormel development is classified into 5 stages. I: stolons before swelling of the tips; II: pale cormels $(\Phi=0-5 \mathrm{~mm})$; III: cormels that have turned yellow $(\Phi=$ 5-7 mm); IV: enlarged cormels $(\Phi=7-9 \mathrm{~mm})$; cormels, as planting stocks $(\Phi=9-11 \mathrm{~mm})$. White bars $=1 \mathrm{~mm}$, and yellow bars $=1 \mathrm{~cm}$; $\mathbf{B}$ Sections of stolons and cormels at different stages stained by PAS. Va: vascular cells; pa: parenchyma cells. Black bars $=100 \mu \mathrm{m}$, red bars $=50 \mu \mathrm{m}$. Starch (C) and sucrose (D) levels at the different cormel development stages (I-V). Endogenous ABA (E) and $\mathrm{GA}_{3}(\mathbf{F})$ levels at the different cormel development stages $(I-V$ ). Averages of three biological replicates \pm SDs (cormels/stolons from three different plants per biological replicate) are shown. Different letters represent statistically significant differences at $p<0.05$ (one-way ANOVA and Tukey HSD post hoc test)

stocks in commercial cultivation (V; approximately $1 \mathrm{~cm}$ in diameter). To characterize the different stages of cormel development (I-V), we observed cells and sugar distribution by periodic acid-Schiff (PAS) staining. The results showed that parenchyma cells and vascular cells in stolons (stage I; Fig. 1B) accumulated or transported fewer sugars than formed cormels (stages II-V). After cormels formed at the tips of stolons, parenchyma cells started to accumulate sugars that were delivered from the mother corm (Fig. 1B). The distribution of sugars in cormels was not equal, with the bottom of cormels accumulating more sugars (Fig. 1B). At later stages (IV to V), the parenchyma cells were much larger, and the vascular cells accumulated sugars at the tip (Fig. 1B).

Next, we analyzed the starch and sucrose levels at the different developmental stages. The levels of both starch and sucrose were low in stolons but increased gradually in cormels from stage II to V (Fig. 1C, D).

As phytohormones regulate plant development, including germination, vegetative development, and reproductive development ${ }^{36}$, we quantified phytohormone levels during cormel development. When corms formed at the tips of cormels (transition from I to II), the endogenous $A B A$ level increased sharply, while the $\mathrm{GA}_{3}$ level decreased (Fig. 1E-F). When cormels developed from
III to $\mathrm{V}$, endogenous ABA was maintained at a relatively high level (although not significantly higher than the level at stage I), while $\mathrm{GA}_{3}$ remained at a relatively stable lower level than the level at stage I (Fig. 1E, F). These results indicate a dramatic increase in sucrose and starch levels during corm development, accompanied by a transient increase in the ABA level and a decrease in the GA level.

\section{Exogenous $A B A$ and GA affect the development of Gladiolus corm and cormels}

Given the opposite changes in $\mathrm{ABA}$ and $\mathrm{GA}_{3}$ levels during the transition from stage I to II (Fig. 1E, F), we hypothesized that these hormones play opposite roles in Gladiolus cormel formation and enlargement. To test our hypothesis, we treated plants with $\mathrm{ABA}$ and $\mathrm{GA}_{3}$ at 10 weeks after planting (WAP). Usually, cormels are largely formed at 14 WAP. After 6 weeks of treatment, plants under ABA treatment had larger mother corms (Fig. 2A-C). Moreover, ABA also significantly promoted the formation and development of cormels, resulting in more cormels and higher yield (Fig. 2D, E). Plants subjected to $\mathrm{GA}_{3}$ treatment had a slightly smaller circumference, but their corms were much heavier (Fig. 2BC). $\mathrm{GA}_{3}$ played a negative role in corm formation by reducing the number of cormels (Fig. 2D). Taken 

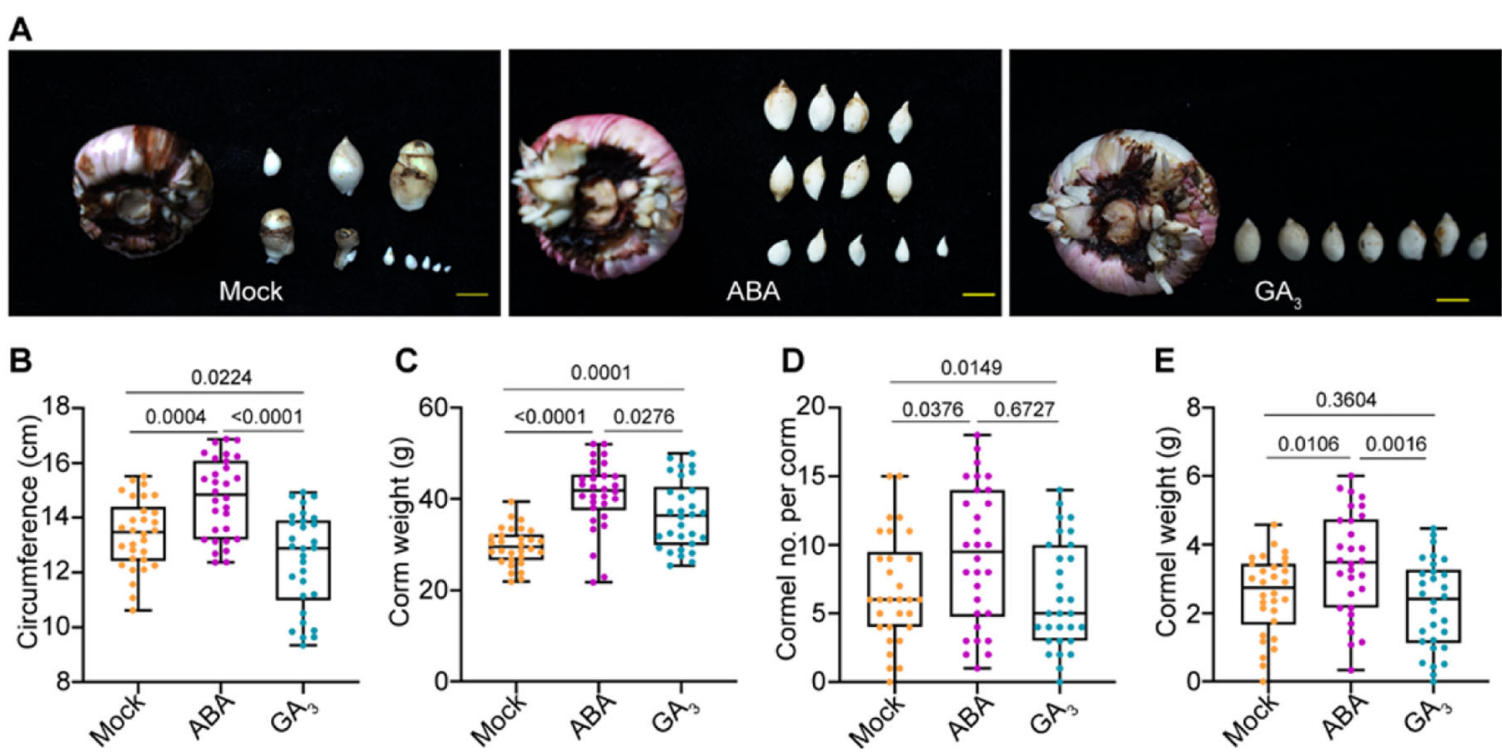

Fig. 2 Gladiolus corm and cormel development was affected by ABA and $\mathbf{G A}_{\mathbf{3}}$. A Phenotypes of corm and cormels after 6 weeks of treatment with $\mathrm{ABA}(0.5 \mathrm{mg} / \mathrm{L})$ and $\mathrm{GA}_{3}(0.5 \mathrm{mg} / \mathrm{L})$. Yellow scale bars $=1 \mathrm{~cm}$. The circumference $(\mathbf{B})$ and weight $(\mathbf{C})$ of mother corms under different treatments. D Cormel numbers per plant under different treatments. E Total cormel yield per plant under different treatments. The data were collected after 6 weeks of treatment. The experiments were performed with three biological replicates (10 corms per biological replicate). The significant differences between the mock and treatments were determined by Tukey's multiple comparison test. The $P$ value is indicated above the black line

together, the results show that ABA plays a positive role in the formation and development of cormels and corm development, while GA negatively affects the formation of cormels.

\section{Silencing ABA and GA biosynthesis genes affects Gladiolus cormel development}

To further address the role of ABA and GA in cormel development, we silenced ABA and GA biosynthesis genes in cormels. Silencing GhNCED (NINE-CISEPOXYCAROTENOID DIOXYGENASE), a key enzyme in ABA biosynthesis in Gladiolus cormels, led to reduced ABA content (Fig. S1), increased $\mathrm{GA}_{3}$ content (Fig. S1) and early sprouting ${ }^{37}$. Here, we found that silencing GhNCED in corms before planting also reduced the starch content in leaves (Fig. 3A) and led to the generation of smaller and lighter corms (Fig. 3B-D). Conversely, silencing the GA biosynthesis gene GhGA20ox decreased $\mathrm{GA}_{3}$ levels and had the opposite effect, promoting the development of enlarged and heavier corms (Fig. 3B-D; Fig. S1). Furthermore, the starch content in GhNCEDsilenced corms was dramatically lower than that in the control, while it was much higher in GhGA20ox ( $\mathrm{gib}$ berellin 20-oxidase)-silenced corms (Fig. 3A, E, F). The sucrose levels did not change as obviously as those of starch in the silenced corms. These results, taken together with the previous results (Figs. 1 and 2), led us to conclude that ABA has positive effects, while GA has negative effects, on corm development. The effect of ABA and GA on corm development may be correlated with starch.

\section{Expression of GhSUS2 is correlated with Gladiolus corm development and regulated by $A B A$ and $G A$}

Previous work has shown that SUSy, not INV, is the dominant active enzyme in actively growing sink organs in geophytes, such as potato tuber and cassava roots $^{19,38,39}$. Moreover, SUSy is essential for sink strength, especially in starch-accumulating organs ${ }^{19}$. To identify corm-expressed GhSUSs, we screened our cormel transcriptome database ${ }^{40}$. We found six unigenes expressed in cormels, of which GlaUn069031 was the most abundant (Fig. S2A). GlaUn069031 has a sequence similar to those of the homologous genes in Arabidopsis (AtSUS2) and rice (OsSUS2), so we named it GhSUS2 (Fig. S3A). GhSUS2 shared a conserved Ser-phosphorylation site at the N-terminus of the amino acid sequence (Fig. S3B).

Given that ABA and GA affected starch synthesis in Gladiolus (Fig. 3), we investigated the relationship between these two hormones and GhSUS2. First, we explored the expression pattern of GhSUS2 in corms treated with $\mathrm{ABA}$ and $\mathrm{GA}_{3}$. Quantitative RT-PCR results revealed that GhSUS2 expression was induced by ABA and slightly repressed by $\mathrm{GA}_{3}$, although the change was not statistically significant (Fig. 4A). We then tested the transcript level of GhSUS2 in GhNCED- and GhGA20oxsilenced corms. The results showed that GhSUS2 expression was decreased in GhNCED-silenced corms but 

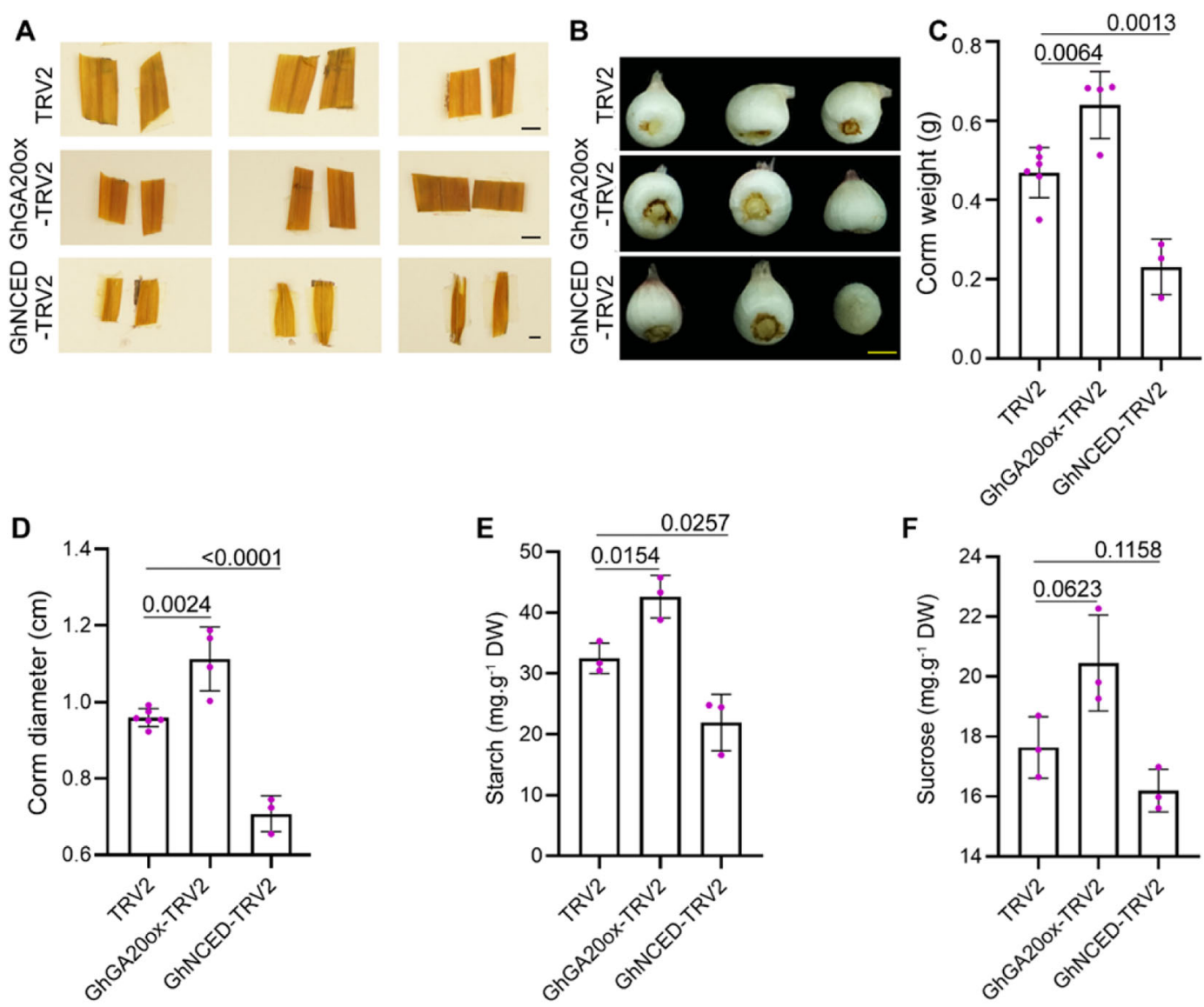

Fig. 3 GhNCED and GhGA20ox regulate corm development and starch biosynthesis. A. lodine staining of 2-month-old gene-silenced leaves. A darker color indicates a higher level of starch. Bars $=1 \mathrm{~cm}$. B. Silencing of GhNCED or GhGA200x affected corm development. The image was taken 4 months after planting. Bars $=0.5 \mathrm{~cm}$. Measurements of corm weight (C) and diameter (D) in 4-month-old silenced plants. E. Silencing of GhNCED or GhGA20ox affected starch content in 4-month-old corms. F. Sucrose content at 4 months of age in gene-silenced corms. Error bars represent the SD of different silenced lines. Significant differences were determined by Tukey's multiple comparison test. The $P$ value is indicated above the black line

increased in GhGA20ox-silenced corms (Fig. 4B). Altogether, our data indicate that GhSUS2 expression is induced by ABA and repressed by GA.

To test the role of GhSUS2 in cormel development, we analyzed its expression pattern in different stages of cormel development $(I-V)$ and in different organs. GhSUS2 was highly expressed in sink organs (corms and cormels), and its expression level gradually increased with cormel development (Fig. 4C, D). In addition, we also tested GhSUS2 expression in cormels at different growth points. The cormel was formed starting from 10 weeks after planting (WAP), developed until 26 WAP, and dried at room temperature for 4 additional weeks. The expression level of GhSUS2 closely matched the level of sucrose accumulation in cormels (Fig. S2B, C).

Plant sucrose synthase isozymes are mainly located in the cytosol or adjacent plasma membrane ${ }^{19}$. To trace the protein localization, GFP was fused in frame with GhSUS2 (pSuper: GFP-GhSUS2). A green fluorescence signal of
GFP-GhSUS2 was observed in the cytosol (Fig. 4E), showing similar subcellular localization as its homologs in potato $^{25}$. This finding suggests that GhSUS2 is a cytosolic SUS.

\section{Silencing of GhSUS2 represses Gladiolus corm development by decreasing starch content}

As the expression of GhSUS2 is correlated with starch synthesis and corm development, we reasoned that corm development should be regulated by GhSUS2. To test our hypothesis, we silenced GhSUS2 in corms before planting them. After four months of growth, the silenced plants were dug out, and the corm size, corm weight, and cormel frequency were measured. Notably, the corm diameter in GhSUS2-silenced plants was significantly smaller than that in the control (Fig. 5A, B). The fresh weight of silenced corms was also smaller than that of the control (Fig. 5C). In addition to its effects on corm development, GhSUS2 regulated cormel formation. The cormel 

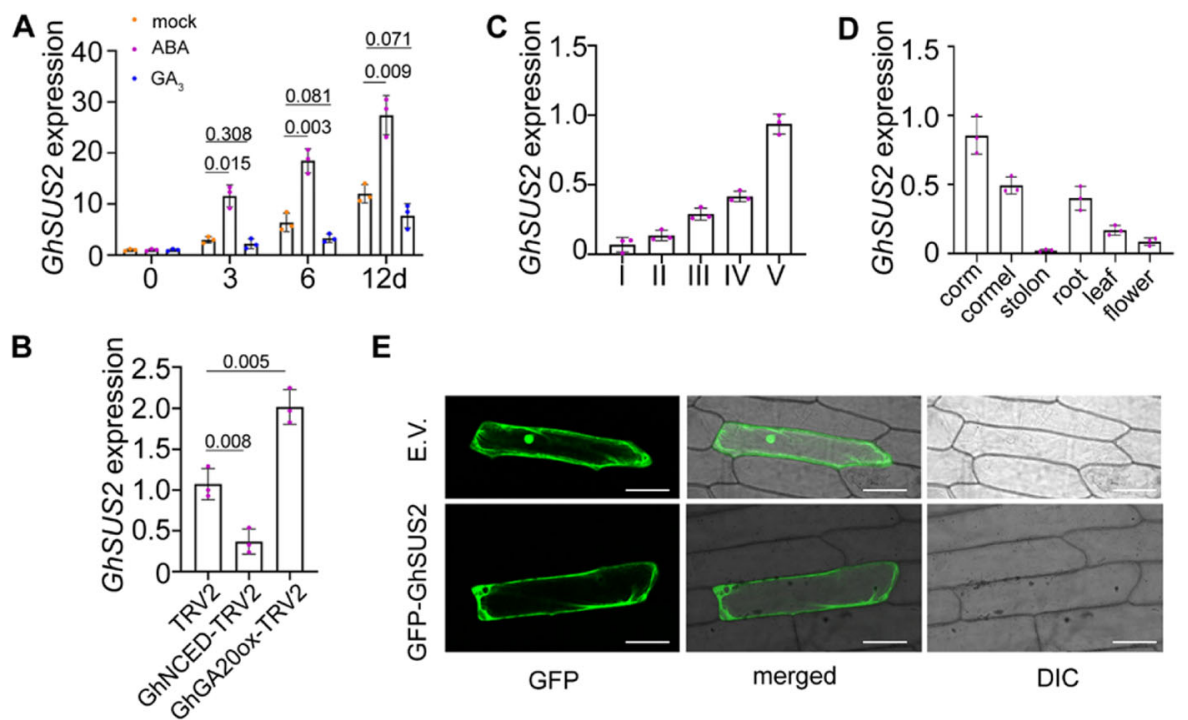

E

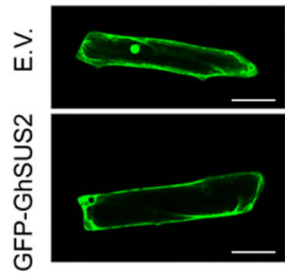

GFP

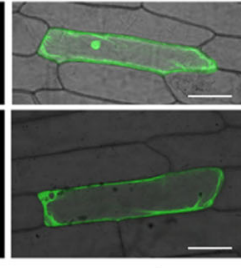

merged

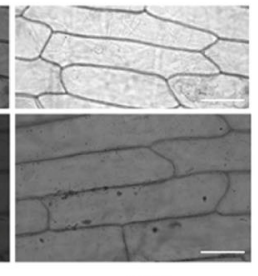

DIC

Fig. 4 Expression of GhSUS2 increases during Gladiolus corm development and is differentially regulated by ABA and GA. A qRT-PCR showing increased GhSUS2 transcript levels under ABA $(0.5 \mathrm{mg} / \mathrm{L})$ treatment and slightly decreased transcript levels under $\mathrm{GA}_{3}(0.5 \mathrm{mg} / \mathrm{L})$ treatment. B Differential expression of GhSUS2 in GhNCED-TRV2 and GhGA20ox-TRV2 corms. C Increased GhSUS2 transcript levels during corm development. D Expression pattern of GhSUS2 in different organs. Averages of three biological replicates \pm SDs $(n=3)$ are shown. Significant differences were determined by Tukey's multiple comparison test. The $P$ value is indicated above the black line. E Subcellular localization of GFP-GhSUS2 in onion epidermal cells. pSuper: GFP (E.V.; empty vector) was used as the control. Bar $=100 \mu \mathrm{m}$
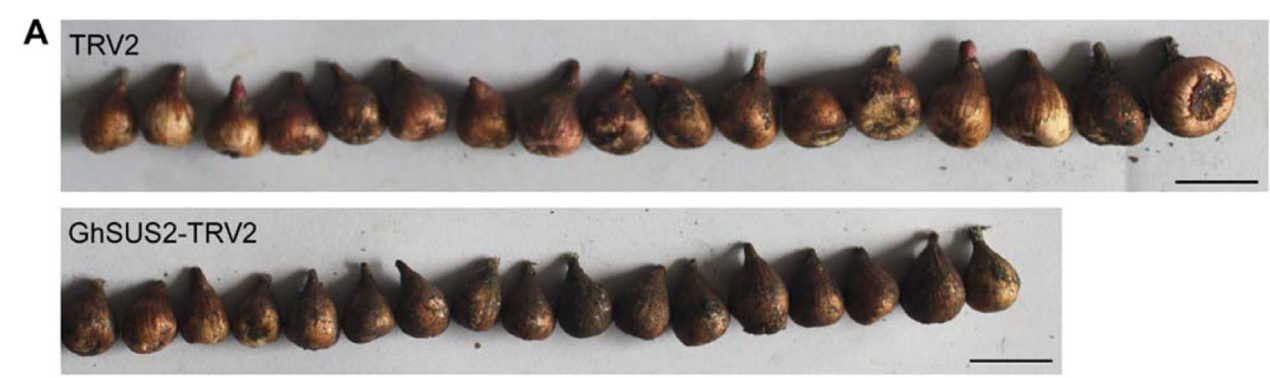

B

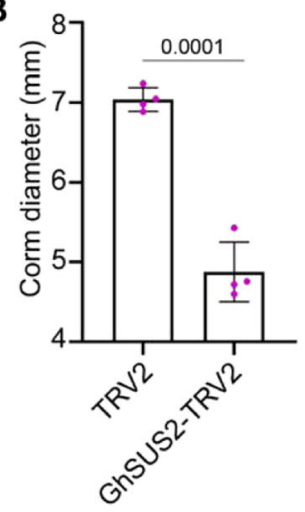

C

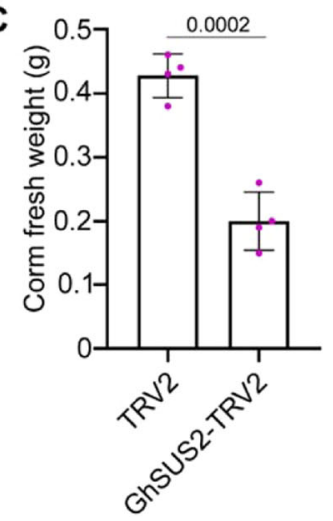

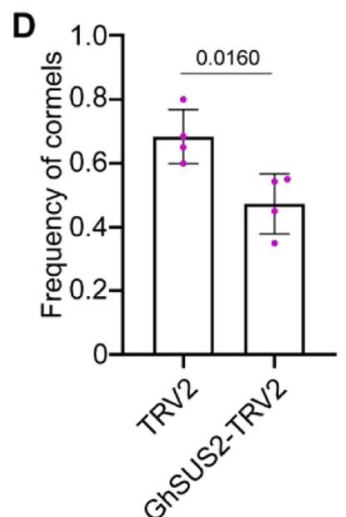

Fig. 5 Silencing of GhSUS2 represses Gladiolus corm development. A Silencing of GhSUS2 in Gladiolus resulted in smaller corms than those observed in the control. The phenotype was observed four months after planting, and 17 representative lines are shown. Black scale bar $=1 \mathrm{~cm}$. Silencing of GhSUS2 in Gladiolus reduced the corm diameter (B) and corm fresh weight (C). D. The cormel frequency in GhSUS2-silenced plants was lower than that in the control. B to $\mathbf{D}$ Averages of four biological repeats, \pm SDs $(n=24)$. Significant differences were determined by the two-sided $t$ test. The $P$ value is indicated above the black line 

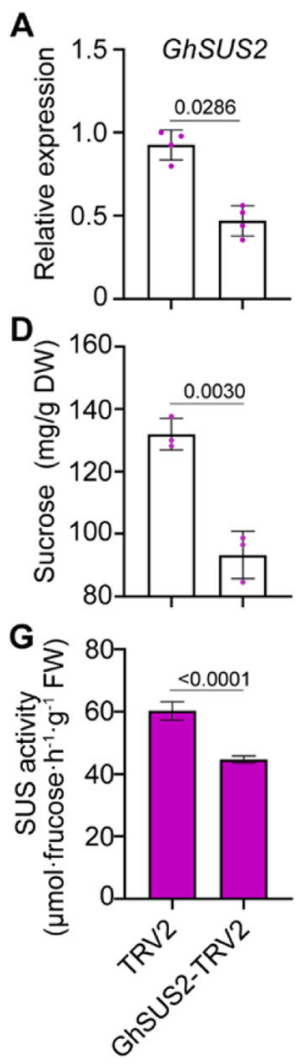

B

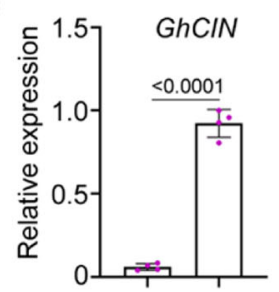

E
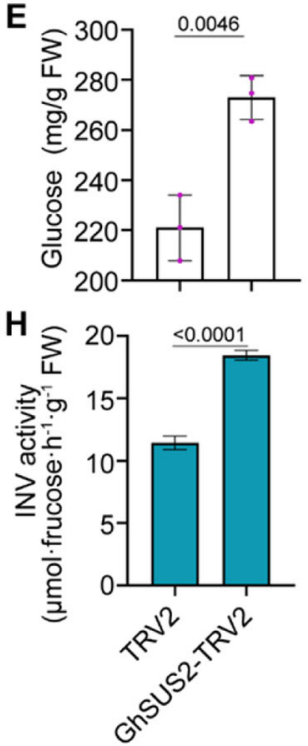

C

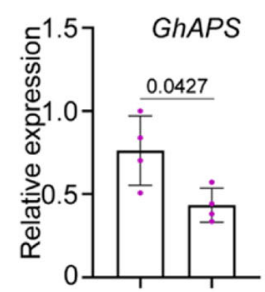

$\mathbf{F}$

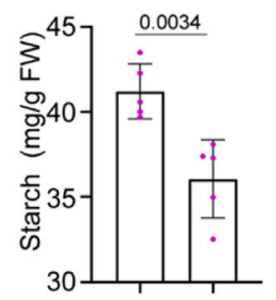

I

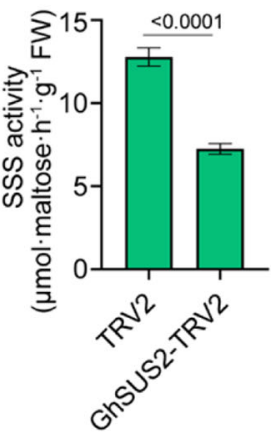

Fig. 6 Silencing of GhSUS2 represses starch synthesis in corms. A Expression of GhSUS2 in silenced corms. B qRT-PCR results showing an increase in the GhCIN transcript level in GhSUS2-silenced corms. C Downregulation of GhAPS expression in GhSUS2-silenced corms. D Decreased sucrose content in GhSUS2-silenced corms. E Accumulation of glucose in GhSUS2-silenced corms. F Repression of starch synthesis in GhSUS2-silenced corms. The enzyme activity of sucrose synthase (G) or soluble starch synthase (SSS; I) was decreased in GhSUS2-silenced corms. $\mathbf{H}$ The enzyme activity of invertase was increased in GhSUS2-silenced corms. Averages of three to five biological replicates \pm SDs are shown. Significant differences were determined by the two-sided $t$-test. The $P$ value is indicated above the black line

frequency in the silenced plants was lower than that in the control (Fig. 5D).

To better understand the role of GhSUS2 in corm development, we determined the expression pattern of key genes in starch biosynthesis in GhSUS2-silenced corms (Fig. 6A). Cytosolic invertase degrades imported sucrose to glucose and fructose in the cytosol ${ }^{41}$. Here, we found that the transcript level of GhCIN was sharply increased in the silenced corms, and accordingly, invertase activity was increased (Fig. 6B, H). The glucose content was higher in the GhSUS2-silenced corms, but the sucrose content was lower (Fig. 6D, E). In previous research, GhAPS has been shown to play a positive role in regulating corm development and starch synthesis ${ }^{33}$. qRT-PCR showed that GhAPS was downregulated when GhSUS2 was silenced (Fig. 6C). Additionally, both sucrose synthase and soluble starch synthase had lower activity in GhSUS2-silenced corms than in the control (Fig. 6G, I). For these reasons, the starch content in GhSUS2-silenced corms was lower than that in the control (Fig. 6F). Overall, we concluded that GhSUS2 promotes corm development by modulating starch synthesis.

\section{Discussion}

Starch serves as the main storage compound in higher plants and accumulates in sink organs (e.g., seeds, bulbs, roots, and buds) before the end of each life cycle. Transcriptome analysis in potato and Lilium suggests that sucrose and starch metabolism is involved in the tuberization and the shoot-to-bulblet transition ${ }^{13,20,42-44}$. In temperate zones, most plants enter dormancy during winter. ABA promotes seed dormancy, bud dormancy and bulb dormancy. Indeed, the ABA level gradually increases during the development of dormant organs (before they become dormant $)^{45-47}$. However, the link between ABA and starch at this developmental stage is less well understood. Here, we found that ABA plays a positive role in corm development and cormel development. While ABA promotes starch synthesis by upregulating GhSUS2 expression, GA has the opposite effect. Furthermore, we showed that starch synthesis positively regulates corm 


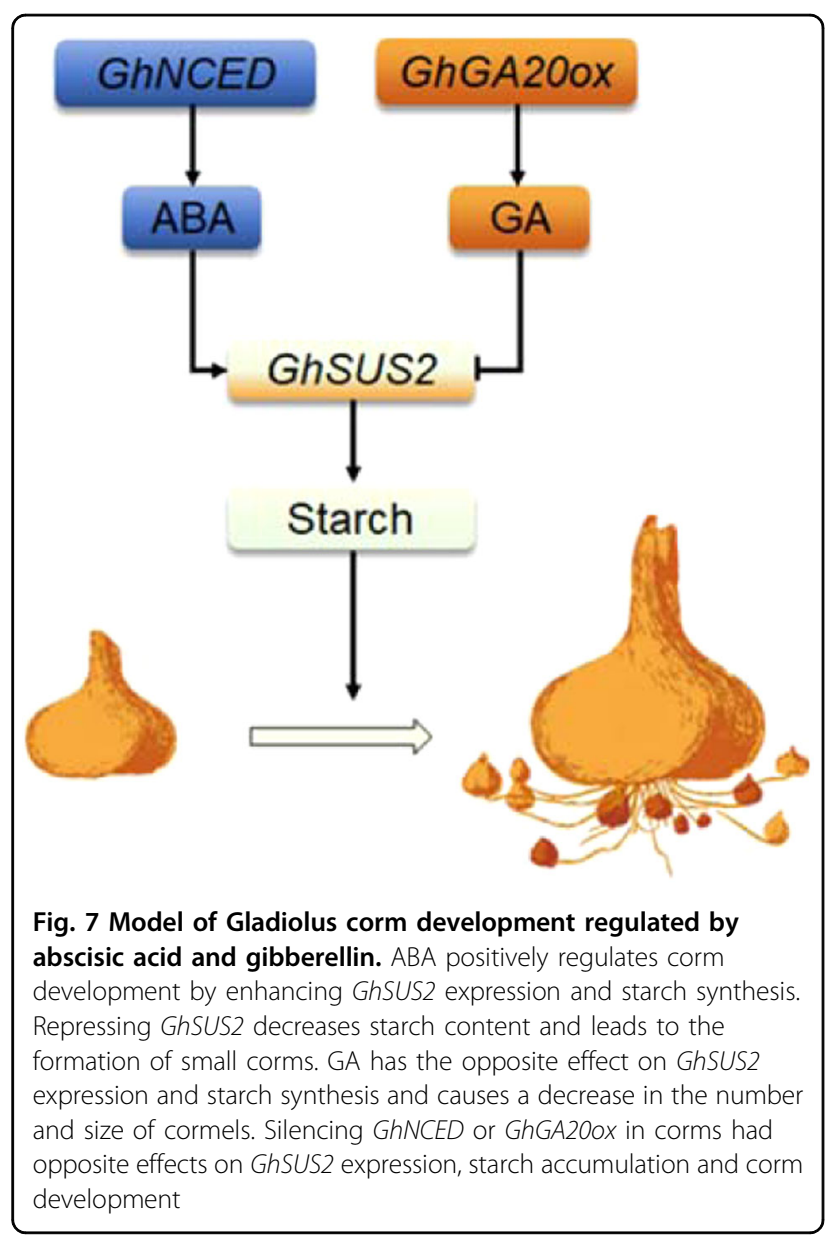

development. We propose that ABA promotes the development of corms in Gladiolus by increasing starch synthesis through upregulation of GhSUS2, while GA has the opposite effect (Fig. 7).

$\mathrm{ABA}$ and GA are two classic hormones in plants that antagonistically regulate several plant developmental processes, including seed maturation, seed dormancy and germination, primary root growth, and flowering time ${ }^{48-50}$. However, GA does not always function as an antagonistic hormone to ABA, e.g., GA inhibits corm dormancy release in the early stage of Gladiolus dormancy release, which is similar to the role of $\mathrm{ABA}^{45,51}$. Here, we provide evidence that $\mathrm{ABA}$ and GA antagonize corm development in Gladiolus: (i) during the transition from stolon to cormel, ABA production was induced and GA production was decreased (Fig. 1); (ii) opposite phenotypes of corm circumference and cormel numbers were observed following treatment with ABA or GA (Fig. 2); (iii) silencing GhNCED or GhGA20ox had opposite effects on corm development (Fig. 3). Notably, we found that $\mathrm{GA}_{3}$ could increase corm weight and the uniformity of cormels (Fig. 3). This might have been caused by cell expansion under GA treatment ${ }^{52}$. GA was shown to promote stolon elongation and reduce the activity of ADP-
$\mathrm{G}^{1,53}$. Moreover, GA could also regulate tuber formation by mediating the FT/CO pathway ${ }^{3}$. However, JA and ABA do not seem to be effective hormones in tuberization ${ }^{1}$. In a recent study, ectopic expression of AtABF4 (ABRE BINDING FACTOR 4 in Arabidopsis) in potato caused GAdefective phenotypes and significantly increased the number and weight of the tubers obtained, suggesting that ABA/ GA cross-talk may be involved in tuberization ${ }^{6}$. Although potato tuberization is regulated by the crosstalk between $\mathrm{ABA}$ and $\mathrm{GA}$, the effects of ABA are always mediated by a change in the levels of GA, which is ultimately the master regulator of tuberization ${ }^{6,54,55}$. Previously, JA was reported to promote corm expansion in Gladiolus ${ }^{35}$, suggesting that the effects of hormones may be species dependent.

In this study, we showed that ABA increases the starch content in corms and promotes corm development and stolon-to-cormel transition. Typically, discourse on tuber development revolves around gibberellins. In the regulation of Gladiolus corm development and starch/sucrose levels, the effect of ABA was more obvious than that of $\mathrm{GA}$, suggesting that ABA plays a more prominent role in corm development. GhNCED is most abundant in the stolon at the flowering/blooming stage when corms start to emerge ${ }^{37}$. This is in agreement with the fact that during the cormel transition, the levels of ABA and starch sharply increased (Fig. 1). NCED is a key enzyme in ABA biosynthesis and is widely involved in plant development and abiotic stress $^{56,57}$. Unlike its role in model plants such as Arabidopsis, rice, and tomato, the role of NCED in modified organs is less well known. Here, we characterized the role of GhNCED in sink organs (corm and cormels) and starch synthesis (Fig. 3). Moreover, we found roles for ABA in promoting starch accumulation after stolon swelling, in cormel transition and in corm dormancy $^{37,45}$.

\section{GhSUS2 positively regulates corm development}

Sucrose synthase was discovered in beets, sweet sorghum, and pea seeds in $1955^{58}$. Most SUS members are found in the cytosol, and some are associated with the plasma membrane. A few SUS isoforms are found in the cell wall and other organelles (e.g., vacuole membrane, cytoskeleton, mitochondria, and Golgi apparatus) ${ }^{19,59-62}$. SUS is the primary active enzyme involved in the breakdown of sucrose in sink organs, enhancement of sink strength, and active growth of sink organs ${ }^{19}$. SUS is highly expressed in storage organs such as seeds, fruits, and taproots and is often positively correlated with starch content and fruit size $e^{63-65}$. In potato, reducing SUS by an antisense technique markedly decreased the starch content and tuber yield ${ }^{63}$. Here, we found that GhSUS2 is localized in the cytosol and that the expression of GhSUS2 is positively related to the early developmental stage of cormels and starch accumulation (Fig. 1C, D, E; Fig. S2B). 
Silencing of GhSUS2 in corms resulted in lower levels of GhAPS, sucrose and starch and reduced SSS enzyme activity (Fig. 6). Intriguingly, we found that INV activity and glucose content were increased in GhSUS2-silenced corms (Fig. 6). As SUS activity was reduced in the silenced corm, INV might have taken over sucrose degradation, resulting in increased glucose level. It has been shown that a high concentration of sucrose can induce tuberization in potato and bulb formation in onion ${ }^{7,66}$. In our GhSUS2silenced corms, the sucrose level was also lower than that in the control (Fig. 6). Moreover, ectopic expression of GhSUS2 in Arabidopsis could promote root elongation on sucrose-containing media (Fig. S4), suggesting that GhSUS2 could accelerate sucrose degradation in cells. In GhSUS2-silenced corms, there was a small decrease in starch content, suggesting that silencing GhSUS2 may not only reduce starch biosynthesis but also slow starch degradation or conversion. Given this evidence, we speculate that GhSUS2 is involved in degrading sucrose in the cytosol and in converting sucrose to starch to promote the sucrose flow from source organs to sink organs.

In plants, the relationship between $\mathrm{ABA}$ and sucrose synthase is variable depending on the species. In pea, ABA induces a decline in nitrogen fixation in a manner that is independent of SUS ${ }^{67}$. In rice, ABA treatment shows a positive relationship between SUS and grain filling ${ }^{68}$. How does ABA relate to SUS in Gladiolus corm development? We utilized both exogenous ABA treatment and silencing of the ABA synthesis gene GhNCED to investigate the effects of ABA. Both assays showed that ABA increases the transcription of GhSUS2 and starch in corms. Recently, a bZIP family member, AtABF4, was demonstrated to increase tuber yield through ABA-GA crosstalk regulation ${ }^{49}$. It will be interesting to investigate how $A B A$ signaling-related transcription factors are involved in regulating GhSUS2 in Gladiolus in future work.

In conclusion, we showed that GhSUS2, an essential enzyme in the starch biosynthesis pathway that mediates the antagonism of $\mathrm{ABA}$ and $\mathrm{GA}$, plays an important role in corm development in Gladiolus.

\section{Material and methods}

\section{Plant materials and treatments}

The Gladiolus cultivar 'Rose Supreme' was planted in the Science Research Garden at China Agricultural University. For tracking corm development, stolons and cormels $(\Phi=0-5,5-7,7-9,9-11 \mathrm{~mm})$ were sampled at 10 WAP. For exogenous hormone treatment, uniform corms $(6 \mathrm{~cm}$ in circumference and 3.0-3.8 g) from plants at 10 WAP were irrigated with ABA $(0.5 \mathrm{mg} / \mathrm{L}$; Solarbio, Beijing, China), $\mathrm{GA}_{3}$ (0.5 mg/L; Solarbio, Beijing, China), or water (the control) every 3 days. The plants were lifted after six weeks of treatment. Three biological replicates were examined (10 corms per biological replicate). For counting cormels, the expanded stolons, which were empty inside and not real cormels, were not included.

\section{Measurement of starch, glucose, and sucrose}

Gladiolus corms or stolons $(50 \mathrm{mg}$ ) were ground in powder by liquid nitrogen. The extraction and quantification of starch, glucose, and sucrose were performed as described by Fan et al. ${ }^{69}$.

\section{Measurement of $\mathrm{ABA}$ and $\mathrm{GA}_{3}$}

Gladiolus corms or stolons were collected $(50 \mathrm{mg})$ and ground to a powder with liquid nitrogen. The extraction procedure was performed as described by Wu et al. ${ }^{37} \cdot \mathrm{d}_{6^{-}}$ ABA and $\mathrm{d}_{2}-\mathrm{GA}_{3}$ were used as internal standards. The extracts were analyzed by HPLC-MS/MS ${ }^{70}$. Three biological replicates were examined.

\section{PAS staining}

Gladiolus corms and stolons were fixed in formalinaceto-alcohol (FAA) solution. The dehydration and embedding procedures were performed according to a previously published protocol ${ }^{71}$. Paraffin sections $(8 \mu \mathrm{m})$ were obtained using a slicing machine. The slides were stained with $\mathrm{PAS}^{72}$ and photographed by a light microscope (Olympus BX51, Tokyo, Japan). Sugars were stained in red by PAS.

\section{Virus-induced gene silencing in Gladiolus}

Silencing of the target gene (GhSUS2, GhNCED, or GhGA20ox) by VIGS was performed as described by Zhong et al. ${ }^{73}$ with some modifications. Briefly, a 200-500 base pair (bp) fragment specific to the target gene was generated and cloned into the PTRV2 vector (primers are listed in Table S1). The TRV1, TRV2, and TRV2 target genes were transformed individually into the Agrobacterium GV3101 strain. The transformed colonies were cultured overnight in LB medium containing $50 \mathrm{mg} / \mathrm{L}$ kanamycin and $50 \mathrm{mg} / \mathrm{L}$ rifampicin. Then, bacteria were collected and resuspended in infiltration buffer $(10 \mathrm{mM}$ $\mathrm{MgCl}_{2}, 200 \mathrm{mM}$ acetosyringone, and $10 \mathrm{mM}$ 2-(N-morpholino) ethanesulfonic acid ( $\mathrm{pH}$ 5.6)) to a final $\mathrm{OD}_{600}$ of 1.8. Equal volumes of TRV1 and TRV2 (the control), as well as the TRV1 and TRV2 target genes, were mixed together and kept in the dark for $3 \mathrm{~h}$ at $25^{\circ} \mathrm{C}$ before vacuum infiltration. Corms were submerged in infiltration buffer and infiltrated under $0.9 \mathrm{MPa}$ for $30 \mathrm{~min}$. Ultimately, the corms were planted in pots and grown in a green chamber at $22{ }^{\circ} \mathrm{C}$ under $16 / 8 \mathrm{~h} \mathrm{light/dark}$ for four months.

\section{lodine staining of starch}

Gladiolus leaves were submerged in 75\% (v/v) ethanol at $80^{\circ} \mathrm{C}$ until chlorophyll was eliminated. Then, the leaves were washed with $\mathrm{ddH}_{2} \mathrm{O}$ and stained with Lugol's 
solution $\left[0.06 \% \mathrm{I}_{2}(\mathrm{w} / \mathrm{v}), 0.1 \% \mathrm{KI}(\mathrm{w} / \mathrm{v})\right.$, and $\left.4 \mathrm{mM} \mathrm{HCl}\right]$ for $10 \mathrm{~min}$. Finally, the stained leaves were rinsed with $\mathrm{ddH}_{2} \mathrm{O}$ for $15 \mathrm{~min}^{33}$.

\section{RNA extraction and qRT-PCR}

Total RNA from Gladiolus samples was extracted using the Tiangen RNA Extraction Reagent Kit (Tiangen, Beijing, China) and reverse transcribed with the M-MLV Reverse Transcriptase Kit (TaKaRa, Shiga, Japan). Approximately $400 \mathrm{ng}$ of cDNA was used as the template for qRT-PCR and analyzed by using the Applied Biosystems StepOnePlus ${ }^{\mathrm{TM}}$ real-time PCR system with the Takara qRT-PCR kit. The Gladiolus actin gene acted as the reference gene ${ }^{45}$. The PCR procedures used were based on the manufacturer's instructions. All primers are listed in Table S1, and the data were analyzed with the $2^{-}$ $\Delta \Delta \mathrm{T}$ method $^{74}$.

\section{Subcellular localization of GhSUS2-GFP}

The coding sequence of GhSUS2 was cloned into pCAMBIA1300-GFP with the SalI and KpnI restriction sites (pSuper: GFP-GhSUS2). Both the fusion construct (GFP-GhSUS2) and the control (empty vector; GFP) were transiently transformed into onion epidermal cells by particle bombardment. After incubation at $25^{\circ} \mathrm{C}$ in the dark overnight, the cells were visualized using confocal microscopy (Zeiss LSM 710, Baden-Württemberg, Germany; $488 \mathrm{~nm}$ excitation and a $515-535 \mathrm{~nm}$ bandpass filter).

\section{Activities of SUS, INV, and SSS}

Gladiolus corms were extracted by grinding tissue in liquid nitrogen before adding $1 \mathrm{ml}$ of extraction buffer [25 mM HEPES-KOH (pH 7.3), $5 \mathrm{mM}$ ethylenediamine tetraacetic acid (EDTA), $0.1 \%(\mathrm{w} / \mathrm{v})$ polyvinyl pyrrolidone (Mr 4000), $1 \mathrm{mM}$ dithiothreitol, $0.01 \mathrm{mM}$ leupeptin and $1 \mathrm{mM}$ phenylmethylsulfonyl fluoride]. The extraction procedures were performed as described by Fan et al. ${ }^{69}$. The supernatants were used for the determination of cytosolic invertase activity as described by Wang et al. ${ }^{75}$. SUS activity assays, in the direction of sucrose breakdown, were carried out as described by Wang et al. ${ }^{76}$. Starch synthase activity was measured using the Soluble Starch Synthase Activity Assay Kit (Solarbio, Beijing, China).

\footnotetext{
Acknowledgements

This work was funded by the Beijing Natural Science Foundation (grant 6212012 to J.W.), National Natural Science Foundation projects (grant 31701952 to J.W.), Construction of Beijing Science and Technology Innovation and Service Capacity in Top Subjects (CEFF-PXM2019_014207_000032), and 111 Project of the Ministry of Education (B17043). We thank Dr. Si Ma (China Agricultural University) for providing suggestions for this study. We also thank Dr. Sonia Gazzarrini (University of Toronto) for editing this manuscript.
}

\section{Author contributions}

J.L. and D.L. observed the development of corms and performed the endogenous hormone treatments; S.S. and F.Z. performed the GhSUS2 assays;
Y.L., T.Y., and J.L. performed gene silencing; and J.W. conceived the study and wrote the article. All the authors have read and approved the manuscript.

Conflict of interest

The authors declare no competing interest.

Supplementary information The online version contains supplementary material available at https://doi.org/10.1038/s41438-021-00589-w.

Received: 6 December 2020 Revised: 22 March 2021 Accepted: 4 May 2021 Published online: 01 July 2021

\section{References}

1. Jackson, S. D. Multiple signaling pathways control tuber induction in potato. Plant Physiol. 119, 1-8 (1999).

2. Krauss, A. Interaction of nitrogen nutrition, phytohormones, and tuberization. Potato Physiol. 209-230 (1985).

3. Lehretz, G. G., Sonnewald, S., Hornyik, C., Corral, J. M. \& Sonnewald, U. Post-transcriptional regulation of FLOWERING LOCUS T modulates heatdependent source-sink development in potato. Curr. Biol. 29, 1614-1624 (2019).

4. Pantelic, D., Dragicevic, I. C., Rudic, J., Fu, J. M. \& Momcilovic, I. Effects of high temperature on in vitro tuberization and accumulation of stressresponsive proteins in potato. Hortic. Environ. Biotechnol. 59, 315-324 (2018).

5. Sattelmacher, B. \& Marschner, H. Cytokinin activity in stolons and tubers of Solanum tuberosum during the period of tuberization. Physiol. Plant 44,69-72 (1978).

6. Garcia, M. N. M., Stritzler, M. \& Capiati, D. A. Heterologous expression of Arabidopsis ABF4 gene in potato enhances tuberization through ABA-GA crosstalk regulation. Planta 239, 615-631 (2014).

7. Xu, X., van Lammeren, A. A. M., Vermeer, E. \& Vreugdenhil, D. The role of gibberellin, abscisic acid, and sucrose in the regulation of potato tuber formation in vitro. Plant Physiol. 117, 575-584 (1998).

8. Roumeliotis, E., Visser, R. G. \& Bachem, C. W. A crosstalk of auxin and GA during tuber development. Plant Signal Behav. 7, 1360-1363 (2012).

9. Carrera, E., Bou, J., Garcia-Martinez, J. L. \& Prat, S. Changes in GA 20-oxidase gene expression strongly affect stem length, tuber induction and tuber yield of potato plants. Plant J. 22, 247-256 (2000).

10. Bou-Torrent, J., Martinez-Garcia, J. F., Garcia-Martinez, J. L. \& Prat, S. Gibberellin A1 metabolism contributes to the control of photoperiod-mediated tuberization in potato. PLOS ONE 6, e24458 (2011).

11. Koda, Y. et al. Potato tuber-inducing activities of jasmonic acid and relatedcompounds. Phytochemistry 30, 1435-1438 (1991).

12. Tao, G. Q. et al. Promotion of shoot development and tuberisation in potato by expression of a chimaeric cytokinin synthesis gene at normal and elevated $\mathrm{CO}_{2}$ levels. Funct. Plant Biol. 37, 43-54 (2010).

13. Wu, Y. et al. Change in sucrose cleavage pattern and rapid starch accumulation govern lily shoot-to-bulblet transition in vitro. Front Plant Sci. 11, 564713 (2021).

14. Tuncel, A. et al. Cas9-mediated mutagenesis of potato starch-branching enzymes generates a range of tuber starch phenotypes. Plant Biotechnol. J. 17, 2259-2271 (2019)

15. Zhang, $\mathrm{K}$. et al. SH1-dependent maize seed development and starch synthesis via modulating carbohydrate flow and osmotic potential balance. BMC Plant Biol. 20, https://doi.org/10.1186/s12870-020-02478-1 (2020).

16. Kumar, R., Mukherjee, S. \& Ayele, B. T. Molecular aspects of sucrose transport and its metabolism to starch during seed development in wheat: a comprehensive review. Biotechnol. Adv. 36, 954-967 (2018).

17. Zeeman, S. C., Kossmann, J. \& Smith, A. M. Starch: its metabolism, evolution, and biotechnological modification in plants. Annu Rev. Plant Biol. 61, 209-234 (2010).

18. Ma, S., Li, Y. X., Li, X., Sui, X. L. \& Zhang, Z. X. Phloem unloading strategies and mechanisms in crop fruits. J. Plant Growth Regul. 38, 494-500 (2019).

19. Stein, O. \& Granot, D. An overview of sucrose synthases in plants. Front Plant Sci. 10, 95 (2019). 
20. Potato Genome Sequencing, $C$. et al. Genome sequence and analysis of the tuber crop potato. Nature 475, 189-195 (2011).

21. Cao, H. \& Shannon, J. C. Effect of gibberellin on growth, protein secretion, and starch accumulation in maize endosperm suspension cells. J. Plant Growth Regul. 16, 137-140 (1997).

22. Davies, H. \& Viola, R. The effect of gibberellic acid on starch breakdown in sprouting tubers of Solanum tuberosum L. Ann. Bot. 61, 689-693 (1988).

23. Wuriyanghan, $\mathrm{H}$. et al. The ethylene receptor ETR2 delays floral transition and affects starch accumulation in rice. Plant Cell 21, 1473-1494 (2009).

24. Schmolzer, K., Gutmann, A., Diricks, M., Desmet, T. \& Nidetzky, B. Sucrose synthase: a unique glycosyltransferase for biocatalytic glycosylation process development. Biotechnol. Adv. 34, 88-111 (2016)

25. Baroja-Fernandez, E. et al. Sucrose synthase catalyzes the de novo production of ADPglucose linked to starch biosynthesis in heterotrophic tissues of plants. Plant Cell Physiol. 44, 500-509 (2003).

26. Takeda, $\mathrm{H}$. et al. Phosphorylation of rice sucrose synthase isoforms promotes the activity of sucrose degradation. Plant Biotechnol.-Nar. 34, 107-113 (2017).

27. Xu, S. M., Brill, E., Llewellyn, D. J., Furbank, R. T. \& Ruan, Y. L. Overexpression of a potato sucrose synthase gene in cotton accelerates leaf expansion, reduces seed abortion, and enhances fiber production. Mol. Plant 5, 430-441 (2012).

28. Xu, F. \& Joshi, C. P. Overexpression of aspen sucrose synthase gene promotes growth and development of transgenic Arabidopsis plants. Adv. Biosci. Biotechnol. 1, 426 (2010).

29. Fukao, T. \& Bailey-Serres, J. Plant responses to hypoxia - is survival a balancing act? Trends Plant Sci. 9, 449-456 (2004).

30. Zrenner, R., Salanoubat, M., Willmitzer, L. \& Sonnewald, U. Evidence of the crucial role of sucrose synthase for sink strength using transgenic potato plants (Solanum tuberosum L.). Plant J. 7, 97-107 (1995).

31. Le Nard, M. The physiology of flower bulbs: a comprehensive treatise on the physiology and utilization of ornamental flowering bulbous and tuberous plants (Elsevier, 1993).

32. He, X. L. et al. Effects of lipoxygenase on the corm formation and enlargement in Gladiolus hybridus. Sci. Hortic.-Amst. 118, 60-69 (2008).

33. Seng, S. S. et al. ADP-glucose pyrophosphorylase gene plays a key role in the quality of corm and yield of cormels in gladiolus. Biochem. Bioph. Res. Co. 474, 206-212 (2016).

34. Seng, S. S. et al. Silencing GhAGPL1 reduces the quality and quantity of corms and cormels in gladiolus. J. Am. Soc. Hortic. Sci. 142, 119-U116 (2017).

35. Lian, Q. L. et al. Cloning, characterization and expression analysis of a 9lipoxygenase gene in Gladiolus hybridus. Sci. Hortic.-Amst. 130, 468-475 (2011).

36. Davies, P. J. Plant hormones and their role in plant growth and development (Springer Science \& Business Media, 2012).

37. Wu, J. et al. GhTCP19 transcription factor regulates corm dormancy release by repressing GhNCED expression in gladiolus. Plant Cell Physiol. 60, 52-62 (2019).

38. Morrell, S. \& Rees, T. A. Sugar Metabolism in Developing Tubers of SolanumTuberosum. Phytochemistry 25, 1579-1585 (1986).

39. Sung, S. J. S., Xu, D. P. \& Black, C. C. Identification of actively filling sucrose sinks. Plant Physiol. 89, 1117-1121 (1989).

40. $W u$, J. et al. GhNAC83 inhibits corm dormancy release by regulating ABA signaling and cytokinin biosynthesis in Gladiolus hybridus. J. Exp. Bot. 70, 1221-1237 (2019).

41. Ruan, Y. L. Sucrose metabolism: gateway to diverse carbon use and sugar signaling. Annu Rev. Plant Biol. 65, 33-67 (2014).

42. Li, X. et al. Transcriptome analysis of carbohydrate metabolism during bulblet formation and development in Lilium davidii var. unicolor. Bmc Plant Biol. 14, 358 (2014).

43. Yang, P. et al. Histological and transcriptomic analysis during bulbil formation in Lilium lancifolium. Front Plant Sci. 8, 1508 (2017).

44. Gu, J., Zeng, Z., Wang, Y. \& Lyu, Y. Transcriptome analysis of carbohydrate metabolism genes and molecular regulation of sucrose transport gene LOSUT on the flowering process of developing oriental hybrid lily 'Sorbonne' Bulb. Int. J. Mol. Sci. 21, https://doi.org/10.3390/ijms21093092 (2020).

45. Wu, J. et al. Gladiolus hybridus ABSCISIC ACID INSENSITIVE 5 (GhABI5) is an important transcription factor in $\mathrm{ABA}$ signaling that can enhance Gladiolus corm dormancy and Arabidopsis seed dormancy. Front. Plant Sci. 6, https:// doi.org/10.3389/fpls.2015.00960 (2015).

46. Nambara, E. et al. Abscisic acid and the control of seed dormancy and germination. Seed Sci. Res. 20, 55-67 (2010).

47. Tylewicz, S. et al. Photoperiodic control of seasonal growth is mediated by ABA acting on cell-cell communication. Science 360, 212-214 (2018).
48. S. Yamaguchi, Y., Kamiya, E. Nambara. Regulation of ABA and GA levels during seed development and germination in arabidopsis in seed development dormancy and germination 224-247 (Ann Plant Rev, 2007).

49. Yang, L. et al. ABA-Mediated ROS in Mitochondria Regulate Root Meristem Activity by Controlling PLETHORA Expression in Arabidopsis. Plos Genet 10, https://doi.org/10.1371/journal.pgen.1004791 (2014).

50. Shu, $K$ et al. ABSCISIC ACID-INSENSITIVE 4 negatively regulates flowering through directly promoting Arabidopsis FLOWERING LOCUS C transcription. J. Exp. Bot. 67, 195-205 (2016)

51. Ram, R., Mukherjee, D. \& Manuja, S. Plant growth regulators affect the development of both corms and cormels in Gladiolus. Hort. science 37 , 343-344 (2002).

52. Sanchez-Montesino, $R$. et al. A regulatory module controlling GA-mediated endosperm cell expansion is critical for seed germination in Arabidopsis. Mol. Plant 12, 71-85 (2019)

53. KUMAR, D. \& WAREING, P. F. Factors controlling stolon development in the potato plant. N. Phytol. 71, 639-648 (1972).

54. Muniz Garcia, M. N., Cortelezzi, J. I., Fumagalli, M. \& Capiati, D. A. Expression of the Arabidopsis ABF4 gene in potato increases tuber yield, improves tuber quality and enhances salt and drought tolerance. Plant Mol. Biol. 98, 137-152 (2018).

55. Muniz Garcia, M. N. et al. Characterization of StABF1, a stress-responsive bZIP transcription factor from Solanum tuberosum $L$. that is phosphorylated by StCDPK2 in vitro. Planta 235, 761-778 (2012).

56. Toh, S. et al. High temperature-induced abscisic acid biosynthesis and its role in the inhibition of gibberellin action in Arabidopsis seeds. Plant Physiol. 146, 1368-1385 (2008).

57. Zeevaart, J. A. D. \& Creelman, R. A. Metabolism and physiology of abscisic-acid. Annu Rev. Plant Phys. 39, 439-473 (1988).

58. Leloir, L. F. \& Cardini, C. E. The biosynthesis of sucrose. J. Am. Chem. Soc. 75 6084-6084 (1953).

59. Salnikov, V. V., Grimson, M. J., Seagull, R. W. \& Haigler, C. H. Localization of sucrose synthase and callose in freeze-substituted secondary-wall-stage cotton fibers. Protoplasma 221, 175-184 (2003).

60. Etxeberria, E. \& Gonzalez, P. Evidence for a tonoplast-associated form of sucrose synthase and its potential involvement in sucrose mobilization from the vacuole. J. Exp. Bot. 54, 1407-1414 (2003).

61. Subbaiah, C. C. et al. Mitochondrial localization and putative signaling function of sucrose synthase in maize. J. Biol. Chem. 281, 15625-15635 (2006).

62. Konishi, T., Ohmiya, Y. \& Hayashi, T. Evidence that sucrose loaded into the phloem of a poplar leaf is used directly by sucrose synthase associated with various beta-glucan synthases in the stem. Plant Physiol. 134, 1146-1152 (2004).

63. Herbers, K. \& Sonnewald, U. Molecular determinants of sink strength. Curr. Opin. Plant Biol. 1, 207-216 (1998).

64. Ishimaru, T. et al. Expression patterns of genes encoding carbohydratemetabolizing enzymes and their relationship to grain filling in rice (Oryza sativa L.): comparison of caryopses located at different positions in a panicle. Plant Cell Physiol. 46, 620-628 (2005).

65. Hirose, T., Scofield, G. N. \& Terao, T. An expression analysis profile for the entire sucrose synthase gene family in rice. Plant Sci. 174, 534-543 (2008).

66. Le Guen-Le Saos, F., Hourmant, A., Esnault, F. \& Chauvin, J. E. In vitro bulb development in shallot (Allium cepa L. aggregatum group): Effects of antigibberellins, sucrose and light. Ann. Bot. 89, 419-425 (2002).

67. Gonzalez, E. M., Galvez, L. \& Arrese-lgor, C. Abscisic acid induces a decline in nitrogen fixation that involves leghaemoglobin, but is independent of sucrose synthase activity. J. Exp. Bot. 52, 285-293 (2001).

68. Tang, T., Xie, H., Wang, Y. X., Lu, B. \& Liang, J. S. The effect of sucrose and abscisic acid interaction on sucrose synthase and its relationship to grain filling of rice (Oryza sativa L.). J. Exp. Bot. 60, 2641-2652 (2009).

69. Fan, J. W., Wang, H. Y., Li, X., Sui, X. L. \& Zhang, Z. X. Down-Regulating Cucumber Sucrose Synthase 4 (CSSUS4) Suppresses the Growth and Development of Flowers and Fruits (DEC, 2018). Plant Cell Physiol. 60, 931-931 (2019).

70. Pan, X. Q., Welti, R. \& Wang, X. M. Quantitative analysis of major plant hormones in crude plant extracts by high-performance liquid chromatographymass spectrometry. Nat. Protoc. 5, 986-992 (2010).

71. Sui, J. J. et al. Characterization and functional analysis of transcription factor LoMYB80 related to anther development in Lily (Lilium Oriental Hybrids). J. Plant Growth Regul. 34, 545-557 (2015). 
72. Baum, S. The PAS reaction for staining cell walls. Cold Spring Harbor Protocols 2008, pdb.prot4956 https://doi.org/10.1101/pdb.prot4956 (2008).

73. Zhong, X. H. et al. Virus-induced gene silencing for comparative functional studies in Gladiolus hybridus. Plant Cell Rep. 33, 301-312 (2014)

74. Livak, K. J. \& Schmittgen, T. D. Analysis of relative gene expression data using real-time quantitative $P C R$ and the 2(T)(-Delta Delta C) method. Methods 25 402-408 (2001).
75. Tomlinson, K. L. et al. Evidence that the hexose-to-sucrose ratio does not control the switch to storage product accumulation in oilseeds: analysis of tobacco seed development and effects of overexpressing apoplastic invertase. J. Exp. Bot. 55, 2291-2303 (2004).

76. Wang, $H$. et al. Antisense suppression of cucumber (Cucumis sativus L.) sucrose synthase 3 (CsSUS3) reduces hypoxic stress tolerance. Plant Cell Environ. 37, 795-810 (2014). 